\title{
Detection of serum $\mathrm{AFB}_{1}$-lysine adduct in Malaysia and its association with liver and kidney functions
}

\begin{abstract}
Aflatoxin is ubiquitously found in many foodstuffs and produced by Aspergillus species of fungi. Of many aflatoxin metabolites, $\mathrm{AFB}_{1}$ is classified by the International Agency for Research on Cancer (IARC) as group one carcinogen and linked to the development of hepatocellular carcinoma (HCC). The study on molecular biomarker of aflatoxin provides a better assessment on the extent of human exposure to aflatoxin. In Malaysia, the occurrences of aflatoxin-contaminated foods have been documented, but there is a lack of data on human exposure to aflatoxin. Hence, this study investigated the occurrence of $\mathrm{AFB}_{1^{-}}$ lysine adduct in serum samples and its association with liver and kidney functions. $5 \mathrm{ml}$ fasting blood samples were collected from seventy-one subjects $(n=71)$ for the measurement of $\mathrm{AFB}_{1}$-lysine adduct, albumin, total bilirubin, AST (aspartate aminotransferase), ALT (alanine transaminase), ALP (alkaline phosphatase), GGT (gamma-glutamyl transpeptidase), creatinine and BUN (blood urea nitrogen). The $\mathrm{AFB}_{1}$-lysine adduct was detected in all serum samples (100\% detection rate) with a mean of $6.85 \pm 3.20 \mathrm{pg} / \mathrm{mg}$ albumin (range: $1.13-18.85 \mathrm{pg} / \mathrm{mg}$ albumin). Male subjects (mean: $8.03 \pm 3.41 \mathrm{pg} / \mathrm{mg}$ albumin) had significantly higher adduct levels than female subjects (mean: $5.64 \pm 2.46 \mathrm{pg} / \mathrm{mg}$ albumin) ( $p<0.01)$. It was noteworthy that subjects with adduct levels greater than average ( $>6.85 \mathrm{pg} / \mathrm{mg}$ albumin) had significantly elevated level of total bilirubin $(p<0.01)$, GGT $(p<0.05)$ and creatinine $(p<0.01)$. Nevertheless, only the level of total bilirubin, $(r=0.347, p$-value $=0.003)$ and creatinine $(r=0.318, p$-value $=0.007)$ showed significant and positive correlation with the level of $\mathrm{AFB}_{1}$-lysine adduct. This study provides a valuable insight on human exposure to aflatoxin in Malaysia. Given that aflatoxin can pose serious problem to the health, intervention strategies should be implemented to limit/reduce human exposure to aflatoxin. Besides, a study with a big sample size should be warranted in order to assess aflatoxin exposure in the general population of Malaysia.
\end{abstract}

Keyword: Aflatoxin; $\mathrm{AFB}_{1}$-lysine adduct; Malaysia; Liver and kidney functions 